Service social

\title{
Le groupe au service des familles. Analyse comparative de deux types d'interventions complémentaires
}

\section{Lise Darveau-Fournier et Alice M. Home}

Volume 39, numéro 1, 1990

Le groupe ici et ailleurs

URI : https://id.erudit.org/iderudit/706458ar

DOI : https://doi.org/10.7202/706458ar

Aller au sommaire du numéro

Éditeur(s)

École de service social de l'Université Laval

ISSN

1708-1734 (numérique)

Découvrir la revue

Citer cet article

Darveau-Fournier, L. \& Home, A. M. (1990). Le groupe au service des familles. Analyse comparative de deux types d'interventions complémentaires. Service social, 39(1), 75-96. https://doi.org/10.7202/706458ar
Résumé de l'article

Cet article fait l'analyse comparative de deux interventions de groupe effectuées dans le cadre d'un projet-pilote de formation et de soutien aux familles d'un milieu à « haut risque ». Le projet regroupait des familles-gardiennes et des familles-utilisatrices, et se voulait un moyen de contrer l'isolement et la surcharge ressentis par de nombreuses familles et de favoriser leur implication dans la recherche de réponses novatrices à leurs besoins.

Les objectifs, le programme, le fonctionnement et les résultats de ces groupes sont examinés à la lumière de la théorie et de la pratique du service social des groupes. 
Lise Darveau-Fournier, professeure à l'École de service social, Université Laval.

Alice M. Home, professeure à l'École de service social, Université de Montréal.

\title{
Le groupe au service des familles Analyse comparative de deux types d'interventions complémentaires*
}

\author{
Lise Darveau-Fournier \\ Alice M. Home
}

Les transformations sociales récentes ont rendu la vie familiale de plus en plus difficile. L'affaiblissement des réseaux de soutien familial et communautaire et l'apparition de modèles familiaux pluralistes augmentent le stress qui accompagne le rôle parental, particulièrement chez les familles monoparentales qui vivent dans des conditions socio-économiques défavorables. L'effet cumulatif de la pauvreté tant économique que sociale, les conflits de rôles, les surcharges de travail et le manque de ressources provoquent chez de nombreuses familles des sentiments d'incompétence et d'impuissance (Morrison et al., 1986).

L'intervention sociale individuelle axée sur l'acquisition de compétences parentales s'est avérée une réponse incomplète à leurs besoins car elle ne modifie pas l'environnement social, lequel a pourtant une influence prépondérante sur la qualité des soins offerts aux enfants (Comité de la santé mentale, 1985; Garbarino et Sherman, 1980). L'utilisation de modèles variés d'intervention de groupe peut s'avérer un moyen approprié de contrer l'isolement et la surcharge ressentis par de nombreuses familles et de favoriser leur implication dans la recherche de réponses novatrices à leurs besoins.

Cet article présente une analyse comparative de deux interventions de groupe réalisées dans le cadre d'un projet-pilote de soutien aux familles d'un quartier urbain identifié à haut risque. Nous 
présentons brièvement le cadre général du projet, suivi des principaux appuis théoriques qui sous-tendent l'utilisation des groupes comme mode d'intervention. Nous examinons ensuite les caractéristiques de chacun des groupes concernés : celui des familles gardiennes (FG) et celui des familles utilisatrices (FU). Les méthodes d'évaluation retenues sont décrites à la section suivante. Nous analysons ensuite les résultats obtenus par rapport à la structure et au fonctionnement des deux groupes avant d'aborder ceux reliés à l'atteinte des objectifs et à la satisfaction des membres. Nous terminons en discutant quelques questions qui se dégagent de l'analyse effectuée.

\section{Un service de soutien et d'entraide}

Familles-gardiennes de la Basse-Ville était un projet-pilote de recherche-action qui s'inscrivait dans une double perspective de prévention et de prise en charge communautaire. II s'adressait aux mères de jeunes enfants aux prises avec différents problèmes socioéconomiques et personnels tels que la pauvreté et l'épuisement physique et émotionnel. Ce projet a été développé conjointement par des intervenantes d'un centre local de services communautaires et des femmes du milieu concerné, auxquelles s'est jointe une équipe de chercheuses ${ }^{1}$. Le but principal du projet était de prévenir la détérioration familiale en offrant comme mesure de répit aux parents un service de gardiennage à court terme, facilement et rapidement accessible, chez des familles du quartier. Ce service visait, par la même occasion, à garder les enfants dans leur milieu habituel de vie, prévenant ainsi les perturbations inutiles et les placements institutionnels non nécessaires. Cent soixante et onze familles, comprenant 283 enfants, ont bénéficié du gardiennage à une ou quelques reprises.

Un des aspects innovateurs du projet résidait dans son objectif de prise en charge communautaire. C'est dans cette optique que des rencontres de groupes ont été organisées afin d'assurer soutien et formation aux femmes impliquées : membres du collectif, familles gardiennes et familles utilisatrices.

\section{Les groupes, outils de soutien et de formation}

La théorie du service social des groupes et différentes expériences d'intervention fournissent de nombreux appuis à l'utilisation de diverses formes de groupe à des fins de soutien social et de formation (Lewis, 1983; Gottlieb, 1986; Rosenberg, 1984; Gitterman et Shulman, 1986). Les familles aux prises avec des conditions de vie stressantes ont toujours constitué une clientèle traditionnelle des centres 
communautaires où les travailleuses et travailleurs sociaux de groupe les desservent dans l'esprit du modèle à buts sociaux. Ce dernier reconnaît les racines environnementales des problèmes sociaux, tout en insistant sur la capacité potentielle de chaque individu de s'engager et de participer à des activités de nature à augmenter ses habiletés et sa confiance (Coyle, 1959). Ce modèle s'actualise souvent à travers des groupes de socialisation; ces derniers visent alors à aider les populations marginalisées et isolées à acquérir les habiletés sociales nécessaires à une meilleure intégration dans leur communauté (Klein, 1972). Un programme d'activités incluant des sorties et des loisirs en plus de périodes de discussion, constitue un moyen $d^{\prime}$ atteindre ces objectifs. La participation active des membres et le fait de mettre l'accent sur le processus de groupe sont d'autres caractéristiques de ce type de groupe (Toseland et Rivas, 1984).

Le modèle à buts sociaux s'avère également utile pour former des citoyens et citoyennes qui veulent s'impliquer davantage dans leur communauté. Le groupe devient un lieu privilégié pour le développement d'habiletés et d'autonomie en vue d'une implication dans la prise en charge éventuelle des problèmes communautaires (Lewis, 1983). Les travailleuses et travailleurs sociaux doivent identifier et mobiliser les ressources dans le groupe, savoir quand et comment encourager I'autonomie et l'initiative des leaders naturels, tout en leur offrant soutien et formation (Home, 1983).

À ces principes s'ajoutent certaines innovations théoriques susceptibles d'éclaircir la question sur la manière d'intervenir auprès de familles stressées contemporaines. Le concept de soutien social constitue un des développements théoriques les plus importants de la dernière décennie. II inclut divers types d'aide (aide concrète, information, conseils, aide affective, etc.) offerts par des pairs (Gottlieb, 1983). Le réseau de soutien social est une structure stable qui fournit un appui aux efforts requis pour faire face aux situations de la vie quotidienne (Garbarino, 1986). Le réseau fournit une aide informelle qui peut être complémentaire à l'aide professionnelle ou lui servir d'alternative et qui peut diminuer l'impact des situations stressantes chez les personnes affectées.

II existe plusieurs stratégies pour augmenter la qualité et la quantité du soutien disponible. L'une d'elles consiste à former spécifiquement des personnes pour qu'elles puissent devenir des aidantes et aidants non professionnels. Ce type d'aide peut se réaliser à travers un jumelage entre des individus en situation de besoin et d'autres ayant expérimenté des conditions similaires. Cette stratégie se combine souvent avec la mise sur pied de groupes de soutien/ entraide qui s'adressent autant aux bénéficiaires qu'aux aidantes et aidants non professionnels dans un but de formation (Gottlieb, 1986). 
Les groupes de soutien se distinguent des groupes d'entraide par la présence d'un professionnel ou d'une professionnelle. Les uns et les autres s'adressent aux personnes aux prises avec une situation commune; ils visent à augmenter leur capacité d'affronter le stress par l'utilisation de l'aide mutuelle entre les membres (Home, 1985).

La formation peut se réaliser dans les groupes à buts sociaux ou dans ceux axés sur le soutien/entraide; elle se poursuit aussi dans les groupes d'éducation structurée. Ces groupes visent l'acquisition d'habiletés à résoudre des problèmes ou à traverser des étapes de transition. Ce modèle se distingue du courant central de service social des groupes par son accent sur les activités planifiées à l'avance, sur le rôle central de l'animateur ou animatrice ainsi que par l'importance moindre accordée au processus et à l'évolution du groupe (Papell et Rothman, 1983).

Certaines questions concernant l'intervention dans les communautés à haut risque ont été soulevées par différents auteurs et auteures en service social. Avant de tenter d'y répondre, nous décrivons les principales interventions de groupe réalisées dans le cadre d'un projet qui comporte des éléments des modèles précédemment évoqués.

\section{L'intervention de groupe dans le projet "Familles-gardiennes"}

Différentes interventions de groupe ont été menées dans le but de prolonger et compléter le répit offert par le gardiennage. Trois groupes principaux ont fonctionné dans le cadre du projet-pilote. Le premier, formé des membres du collectif, s'est réuni au moins une fois par semaine; il s'agissait d'un groupe de tâche auquel s'est ajouté un volet de formation à la gestion et à l'animation. Nous ne discuterons pas de ce groupe dans le présent article, l'ayant déjà fait ailleurs (Darveau-Fournier et Home, sous presse). L'analyse que nous présentons dans cet article porte sur le groupe des familles gardiennes (FG) et celui des familles utilisatrices $(F U)^{2}$. Ces groupes sont complémentaires parce que tout en s'adressant à deux catégories de familles, avec des objectifs et des moyens spécifiques à chacune, ils visaient tous les deux la réalisation des objectifs globaux du projet. De plus, une des interventions (celle auprès des FG) était de nature à influencer l'autre (celle auprès des FU). Par exemple, le développement $d$ 'habiletés particulières recherchées chez les premières pouvait contribuer à assurer une qualité de relation et de soutien aux deuxièmes et faciliter leur participation aux objectifs du projet; de même, cette participation active des FU et leur collaboration avec les FG pouvaient contribuer à faciliter le rôle de parent substitut de ces 
dernières. Ces deux interventions se complétaient alors mutuellement.

Le groupe de familles gardiennes était un groupe fermé s'adressant à l'ensemble des FG. Le projet en regroupait habituellement entre 8 à 10 à la fois. Vingt et une familles en ont fait partie dont certaines durant plus d'un an. Elles étaient, pour la plupart, biparentales et vivaient dans des conditions socio-économiques plus favorables que la majorité des citoyens de leur quartier : revenu modeste mais régulier, logement convenable. Elles étaient, de plus, reconnues par le collectif comme n'ayant aucun problème personnel ou social majeur et jouissaient $d$ 'une crédibilité dans leur milieu. Ces familles s'étaient jointes volontairement au projet, certaines davantage par goût de s'impliquer dans leur milieu, d'autres plus pour ajouter un complément à leur revenu, mais toutes par amour pour les enfants. Ce sont surtout les femmes qui assumaient la responsabilité du gardiennage mais plusieurs se disaient beaucoup aidées par leur conjoint et leurs enfants. Les principaux buts visés par leur groupe étaient le soutien mutuel, de même que la formation au rôle de parents substituts des enfants gardés et d'aidantes non professionnelles auprès des familles utilisatrices.

Le groupe des familles utilisatrices était un groupe ouvert s'adressant à l'ensemble des 171 familles qui ont fait garder leurs enfants dans le cadre du projet. Ces familles présentaient des caractéristiques très différentes de celles des familles gardiennes. La majorité se composait de mères jeunes (moins de 35 ans) monoparentales avec un ou deux jeunes enfants. Ces familles étaient pauvres, vivant de prestations gouvernementales. Plusieurs disaient ne pouvoir compter sur aucune aide de leur réseau familial ou social pour prendre un répit de leurs tâches parentales; certaines n'avaient encore jamais fait garder leur enfant à l'extérieur de chez elles, en assumant seules la responsabilité à l'année longue. Les réunions de groupe à leur intention ont débuté après 14 mois de fonctionnement du service de gardiennage; elles visaient à diminuer l'isolement des familles et à leur offrir soutien et information.

\section{L'évaluation de l'intervention}

Le devis d'évaluation des interventions de groupe s'inscrivait dans une optique d'évaluation formative adoptée comme approche méthodologique pour l'ensemble du projet. Celle-ci est appropriée aux projets-pilotes entre autres à cause de son caractère de souplesse qui permet de réajuster l'intervention en cours de route (Rutman, 1984). Des données portant sur l'implantation et sur les effets des deux groupes ont ainsi été recueillies à différents moments à l'aide 
d'instruments diversifiés. Le devis d'évaluation est présenté au tableau 1.

Certains des instruments, tel le questionnaire d'évaluation des rencontres FG, étaient utilisés à des fins de réajustement durant le projet alors que d'autres, tel le questionnaire téléphonique, visaient des objectifs d'évaluation sommative. Tous les instruments ont été élaborés spécifiquement pour le projet à partir de recherches traitant de questions similaires. Les schémas d'entrevue de groupe ont été préparés et révisés après consultation avec une experte; tous les autres instruments ont été pré-expérimentés avant la collecte. Ceci a confirmé la pertinence et l'exclusion mutuelle des catégories de la grille d'observation du groupe FG en plus d'établir un accord interjuge presque parfait; un échantillonnage de type "focus» a alors été employé afin de faciliter l'enregistrement des fréquences (Seaburg, 1985). Les instruments s'adressaient à toute la population impliquée, à l'exception du questionnaire d'évaluation des rencontres de FU, qui s'est limité aux participantes à au moins une rencontre dans les sept derniers mois, afin de réduire les distorsions imputables à la mémoire.

L'évaluation formative privilégie une approche inductive. Nous avons procédé par analyse de contenu pour la plupart des données; une analyse de fréquence des comportements observés a été ajoutée dans le cas du groupe de FG.

La méthodologie employée comporte évidemment des avantages et des limites. Le devis retenu a permis la collecte de certaines données détaillées portant sur les groupes et leur signification pour les participantes. Par contre, l'utilisation de l'auto-évaluation de la satisfaction et des effets des groupes ne permet qu'une appréciation globale des résultats.

\section{Résultats et analyse}

Les données recueillies au cours de l'évaluation permettent de faire ressortir les principales tendances de chacun des deux groupes étudiés et, en les examinant à la lumière de la théorie du service social des groupes, de suggérer certaines pistes en vue d'en maximiser les effets. Nous analysons d'abord le fonctionnement des deux groupes pour nous attarder ensuite à leurs résultats en termes d'atteinte des objectifs et de satisfaction des membres.

\section{Structure et fonctionnement}

La description comparative des deux groupes, présentée dans le tableau 2, permet de constater plusieurs différences dans leur 
structure et leur fonctionnement. Nous examinons d'abord I'organisation de ces groupes ainsi que les modèles théoriques qui les soustendent. Nous considérons aussi leurs programmes, le type d'animation et de participation de même que leur évolution respective.

Les besoins et caractéristiques spécifiques des populations visées, et surtout leur rôle dans le projet, ont influencé le choix de l'organisation des groupes. La formule de groupe fermé a été retenue pour les FAMILLES GARDIENNES. Ces dernières, exerçant des fonctions particulières dans le projet auprès des enfants gardés et des parents, avaient besoin de soutien en même temps que d'encadrement et de formation. L'atteinte de tels objectifs exige une certaine stabilité des membres et une continuité dans le programme. Le groupe fermé permet ces conditions et favorise le développement d'une plus grande intimité entre les membres (Hartford, 1971). Le nombre limité et relativement stable des familles gardiennes facilitait aussi le choix de cette formule.

Le groupe de FG s'est appuyé sur deux modèles théoriques principaux : il alliait des éléments du groupe de soutien et du groupe d'éducation. L'influence du groupe de soutien se reflétait particulièrement dans l'importance accordée au partage d'informations, d'expériences et de sentiments, à l'encouragement mutuel, au conseil et à la résolution de problème (Rosenberg, 1984). Les éléments de formation inclus dans le programme s'inspiraient davantage du modèle d'éducation souple décrit par Auerbach (1971) que de celui d'éducation structurée plus formel (Toseland et Rivas, 1984).

Le programme proposé aux familles gardiennes a comporté surtout des discussions sur le vécu du gardiennage et sur l'accomplissement des rôles qui y sont reliés. Ce programme ne peut toutefois être qualifié de formel ni de structuré. Les apprentissages ne se fondaient pas sur des présentations ou sur des expérimentations à caractère didactique; les sujets discutés émergeaient plutôt des préoccupations des membres et de leurs besoins circonstanciels, rejoignant ainsi des éléments du courant central de service social des groupes (Papell et Rothman, 1983). Des échanges informels se poursuivaient aussi entre membres lors des pauses; un goûter était habituellement servi pour faciliter les contacts et l'établissement d'un climat de confiance prérequis au développement du soutien et de l'entraide (Shulman, 1979).

L'observation des rencontres a permis de constater une évolution dans l'animation des réunions, la participation des membres et le développement du groupe. L'animation, assumée au début par des professionnelles, a été prise en charge assez rapidement par la responsable du collectif, qui a développé ses habiletés à travers la formation et la pratique. Plus directive au début, elle a appris à 
TABLEAU 1

Devis d'évaluation

\begin{tabular}{|c|c|c|c|c|c|}
\hline $\begin{array}{l}\text { Groupe } \\
\text { concerné }\end{array}$ & $\begin{array}{c}\text { Objets } \\
\text { d'évaluation }\end{array}$ & $\begin{array}{l}\text { Instruments } \\
\text { utilisés }\end{array}$ & $\begin{array}{l}\text { Contenu des } \\
\text { instruments }\end{array}$ & $\begin{array}{l}\text { Moment de } \\
\text { collecte }\end{array}$ & $\begin{array}{l}\text { Population ou } \\
\text { échantillon }\end{array}$ \\
\hline \multirow[t]{4}{*}{$\begin{array}{l}\text { Familles } \\
\text { gardiennes }\end{array}$} & $\begin{array}{l}\text { Fonctionnement du } \\
\text { groupe (contenu des } \\
\text { échanges, participa- } \\
\text { tion) }\end{array}$ & $\begin{array}{l}\text { - Grille d'observation } \\
\text { remplie par cher- } \\
\text { cheuse } \\
\text { - Procès-verbaux }\end{array}$ & $\begin{array}{l}\text { - Divers compor- } \\
\text { tements (conseils, } \\
\text { information, aide } \\
\text { concrète, etc.) } \\
\text { - Présences, sujets } \\
\text { discutés, décisions } \\
\text { prises, etc. }\end{array}$ & $\begin{array}{l}18 \text { rencontres sur } 24 \text { : } \\
\text { observation de } 2 \\
\text { périodes de } 5 \text { mi- } \\
\text { nutes choisies au } \\
\text { hasard }\end{array}$ & $\begin{array}{l}\text { Toutes les participan- } \\
\text { tes } \\
\mathrm{N} \text { : entre } 7 \text { et } 10 \text { par } \\
\text { rencontre }\end{array}$ \\
\hline & $\begin{array}{l}\text { Adéquation entre be- } \\
\text { soins et programme }\end{array}$ & $\begin{array}{l}\text { - Échange verbal de } \\
\text { groupe }\end{array}$ & $\begin{array}{l}\text { - Contenu, fonction- } \\
\text { nement, satisfac- } \\
\text { tion, résultats }\end{array}$ & $\begin{array}{l}\text { - À la fin de chaque } \\
\text { rencontre }\end{array}$ & $\begin{array}{l}\text { - Toutes les par- } \\
\text { ticipantes }\end{array}$ \\
\hline & et & $\begin{array}{l}\text { - Questionnaire } \\
\text { d'évaluation des } \\
\text { rencontres }\end{array}$ & $\begin{array}{l}\text { - Satisfaction et résul- } \\
\text { tats des rencontres }\end{array}$ & - Après 12 rencontres & $\begin{array}{l}\text { - Toutes les FG } \\
\mathrm{N}: 8 \text { sur } 9\end{array}$ \\
\hline & $\begin{array}{l}\text { Satisfaction des par- } \\
\text { ticipantes }\end{array}$ & $\begin{array}{l}\text { - Schéma d'entrevue } \\
\text { de groupe }\end{array}$ & $\begin{array}{l}\text { - Fonctionnement, } \\
\text { contenu, climat, ef- } \\
\text { fets, satisfaction }\end{array}$ & $\begin{array}{l}- \text { À la fin du } \\
\text { programme }\end{array}$ & $\begin{array}{l}\text { - Toutes les par- } \\
\text { ticipantes } \\
N: 6 \text { sur } 7\end{array}$ \\
\hline
\end{tabular}




\begin{tabular}{|c|c|c|c|c|c|}
\hline $\begin{array}{l}\text { Familles } \\
\text { utilisatrices }\end{array}$ & $\begin{array}{l}\text { Attentes face aux ren- } \\
\text { contres } \\
\text { Contenu des rencon- } \\
\text { tres } \\
\text { Adéquation entre be- } \\
\text { soins et rencontres } \\
\text { Effets perçus par par- } \\
\text { ticipantes } \\
\text { Degré de satisfaction }\end{array}$ & $\begin{array}{l}\text { - Questionnaire écrit } \\
\text { - Procès-verbaux } \\
\text { - Questionnaire télé- } \\
\text { phonique }\end{array}$ & $\begin{array}{l}\text { - Attentes face au } \\
\text { programme, aux at- } \\
\text { titudes des } \\
\text { animatrices, à } \\
\text { I'horaire, etc. } \\
\text { - Présences, sujets } \\
\text { discutés } \\
\text { - Participation, satis- } \\
\text { faction, effets sur } \\
\text { les réseaux }\end{array}$ & $\begin{array}{l}\text { - À la } 1^{\text {re }} \text { rencontre } \\
\text { (scindée en raison } \\
\text { du nombre de par- } \\
\text { ticipantes) } \\
\text { - À chaque rencon- } \\
\text { tre } \\
\text { - À la fin du } \\
\text { programme }\end{array}$ & $\begin{array}{l}\text { - Toutes les par- } \\
\text { ticipantes } \\
N=12 \text { et } N=13 \\
\\
\text { - Toutes les par- } \\
\text { ticipantes } \\
N \text { : entre } 4 \text { et } 13 \\
\text { - Participantes à au } \\
\text { moins } 1 \text { rencontre } \\
\text { durant les } 7 \text { der- } \\
\text { niers mois } \\
N: 22 \text { sur } 26\end{array}$ \\
\hline
\end{tabular}


TABLEAU 2

Portrait comparatif des groupes

\begin{tabular}{|c|c|c|}
\hline & Groupe de familles gardiennes & Groupe de familles utilisatrices \\
\hline Organisation du groupe & - Fermé & - Ouvert \\
\hline Buts & $\begin{array}{l}\text { - Formation aux habiletés de parents substituts et } \\
\text { d'aidantes non professionnelles } \\
\text { - Soutien mutuel }\end{array}$ & $\begin{array}{l}\text { - Diminution de l'isolement } \\
\text { - Information sur habiletés parentales et ressour- } \\
\text { ces communautaires } \\
\text { - Entraide concrète }\end{array}$ \\
\hline Population visée & - L'ensemble des familles gardiennes & - L'ensemble des familles utilisatrices \\
\hline Nombre de rencontres & -24 & -15 \\
\hline Fréquence & - Rencontres mensuelles & - À intervalles irréguliers \\
\hline Animation & $\begin{array}{l}\text { - Premières rencontres : par intervenantes profes- } \\
\text { sionnelles ou chercheuses } \\
\text { - Majorité des rencontres : par responsables du } \\
\text { collectif } \\
\text { - Dernières rencontres : par sous-groupes de } \\
\text { familles gardiennes }\end{array}$ & $\begin{array}{l}\text { - Par intervenantes professionnelles et cher- } \\
\text { cheuses }\end{array}$ \\
\hline $\begin{array}{l}\text { Activités principales du } \\
\text { programme }\end{array}$ & - Discussion & $\begin{array}{l}\text { - Conférence } \\
\text { - Visionnement de vidéo } \\
\text { - Discussion }\end{array}$ \\
\hline Principaux thèmes & $\begin{array}{l}\text { - Comportements particuliers des enfants } \\
\text { (énurésie, colère, ennui, etc.) } \\
\text { - Attitudes face aux familles utilisatrices } \\
\text { - Collaboration avec les ressources (écoles, collec- } \\
\text { tif, etc.) }\end{array}$ & $\begin{array}{l}\text { - Inceste } \\
\text { - Premiers soins aux enfants } \\
\text { - Ressources communautaires (ex. : Centre de } \\
\text { femmes) } \\
\text { - Connaissance de soi }\end{array}$ \\
\hline
\end{tabular}


susciter la discussion avant d'intervenir elle-même ou de suggérer des solutions. Au plan de la participation, on remarque un taux élevé et constant de présences aux rencontres. Les membres se sont impliqués de façon timide au début; graduellement, ils ont acquis plus de confiance en eux-mêmes, se sont exprimés davantage jusqu'à assumer, à leur tour, en sous-groupe, la préparation et l'animation des dernières rencontres.

Le groupe de FG a connu un certain développement au cours de ses 24 rencontres. On peut considérer qu'il était sur le point de devenir un groupe semi-autonome (Lang, 1972). Les membres commençaient à se sentir plus à l'aise dans le groupe, à exprimer davantage leurs opinions, à assumer certaines responsabilités. La stabilité des membres a sans doute contribué au développement de plus en plus régulier et intense de leur implication, les amenant jusqu'au début de la phase du maintien (Henry, 1981).

La formule de groupe ouvert a été privilégiée pour les FAMILLES UTILISATRICES pour différentes raisons dont leur nombre élevé mais surtout leur faible intérêt envers cette forme de participation et même leurs craintes envers les groupes (Breton, 1985; Shulman, 1988). II s'agissait d'un groupe complètement ouvert dans lequel on ne retrouvait aucune norme spécifique de participation : ni modalité d'intégration des nouveaux membres, ni engagement à participer à un certain nombre de rencontres, ni autres conditions (Schopler et Galinsky, 1984). Le groupe ouvert n'exigeant pas d'engagement à long terme, cette formule pouvait paraître moins menaçante pour les familles et exiger de leur part moins d'implication, facilitant ainsi leur participation.

Les objectifs de départ préconisés pour le groupe de FU suggéraient le choix d'un modèle de groupe de socialisation axé sur le développement d'habiletés sociales et relationnelles (Toseland et Rivas, 1984). Toutefois, en cours de réalisation, une priorité a été accordée à la transmission d'information plutôt qu'à l'utilisation du processus de groupe à des fins de développement de cohésion et d'aide mutuelle. On retrouve donc davantage des caractéristiques $d^{\prime}$ un groupe $d^{\prime}$ information, lequel rassemble des participants et participantes qui varient $d^{\prime}$ une rencontre à l'autre en fonction du thème présenté. Ils et elles y cherchent réponse à des questions qui les préoccupent et sont souvent peu intéressés à s'impliquer dans les processus de groupe et à $s^{\prime}$ engager dans des relations interpersonnelles plus intimes.

Les objectifs du groupe de FU étaient plus généraux et concernaient des aspects plus globaux de la situation de familles vulnérables et du rôle parental. Ils se sont reflétés dans le programme offert. Ce dernier a surtout pris la forme de conférences thématiques, portant 
sur des sujets reliés au rôle parental et aux besoins des participantes en tant que femmes.

La priorité plus faible accordée dans le projet aux rencontres de FU s'est traduite par un nombre limité et une fréquence irrégulière des rencontres. L'animation a été surtout prise en charge par des intervenantes sociales et une infirmière, sauf pour les réunions traitant des attentes des participantes, lesquelles ont été animées par une chercheuse formée en service social des groupes.

La participation des familles utilisatrices a été très faible compte tenu du nombre de familles concernées. Les présences aux rencontres ont varié entre 4 et 13 personnes malgré les invitations téléphoniques faites à chacune et auxquelles plusieurs répondaient qu'elles seraient présentes. II ne faut toutefois pas se surprendre $d^{\prime}$ une telle situation. Bon nombre de femmes se sentaient sans doute obligées de dire oui pour éviter de déplaire au collectif ou aux familles gardiennes à qui elles étaient redevables du gardiennage obtenu (Breton, 1985). Les femmes qui ont assisté aux rencontres se sont peu impliquées; le programme ne favorisait d'ailleurs pas le partage intime entre participantes.

Il est difficile de prétendre à un développement du groupe de FU. On peut peut-être le qualifier davantage de collectivité que de groupe puisqu'on n'y retrouve ni buts communs ni phénomènes essentiels de groupe tels que cohésion, autonomie, procédures ou moyens déterminés par le groupe pour atteindre ses objectifs (Lang, 1986:9). L'absence de contrat clair avec les familles utilisatrices au sujet de leur participation à des activités de groupe, en même temps que le type de programme offert, ont contribué à empêcher le développement des processus qu'on retrouve habituellement dans un groupe.

\section{Atteinte des objectifs et participation des membres}

Le tableau-synthèse 3 présente les principaux résultats qui se dégagent par rapport à l'atteinte des objectifs et à la satisfaction des membres. Nous les analysons à la lumière de la théorie du service social des groupes.

Le groupe de FG visait deux objectifs principaux interreliés de soutien/entraide et de formation. L'observation des rencontres a permis de constater certaines manifestations de soutien. Les plus fréquentes ont été le soutien concret tel le partage d'informations sur les ressources communautaires, et la révélation du vécu telles les difficultés causées par certains comportements de l'enfant ou de la mère. Les participantes ont toutefois indiqué qu'elles auraient souhaité recevoir d'autres formes de soutien dont un marrainage pour les nouvelles familles. 
TABLEAU 3

Synthèse des résultats des groupes

\begin{tabular}{|c|c|c|}
\hline & Familles gardiennes & Familles utilisatrices \\
\hline Degré d'atteinte des objectifs & $\begin{array}{l}\text { 1) Par rapport au soutien et à l'entraide } \\
\text { - comportement de soutien concret et partage } \\
\text { du vécu de gardiennage, mais peu de résolu- } \\
\text { tion de problème } \\
\text { - auraient souhaité marrainage lors de leur in- } \\
\text { tégration au projet } \\
\text { 2) Par rapport à la formation } \\
\text { - rôle de parent substitut auprès des enfants : } \\
\text { les rencontres ont aidé mais peu de chan- } \\
\text { gement de comportement } \\
\text { - rôle d'aidant(e) non professionnel(le) : } \\
\text { quelques modifications dans : attitudes face } \\
\text { aux familles utilisatrices, conception du rôle } \\
\text { des familles gardiennes }\end{array}$ & $\begin{array}{l}\text { 1) Par rapport à la diminution de l'isolement } \\
\text { - peu d'impact sur les réseaux des participantes } \\
\text { à l'extérieur des rencontres } \\
\text { 2) Par rapport à l'information } \\
\text { - information utile reçue sur ressources com- } \\
\text { munautaires et sur certaines habiletés paren- } \\
\text { tales } \\
\text { 3) Par rapport à l'entraide concrète } \\
\text { - peu d'évidence d'échanges informels de } \\
\text { services entre participantes }\end{array}$ \\
\hline Degré de satisfaction & $\begin{array}{l}\text { 1) Par rapport au climat } \\
\text { - se sentaient à l'aise pour discuter difficultés } \\
\text { - sentaient que leurs opinions étaient acceptées } \\
\text { - auraient aimé se connaître davantage entre } \\
\text { elles } \\
\text { 2) Par rapport au contenu } \\
\text { - ont pu discuter suffisamment du gardiennage } \\
\text { - auraient aimé plus d'information sur des } \\
\text { façons de procéder face à certains compor- } \\
\text { tements de l'enfant }\end{array}$ & $\begin{array}{l}\text { 1) Par rapport au climat } \\
\text { - satisfaites du climat d'encouragement mutuel } \\
\text { 2) Par rapport au contenu } \\
\text { - satisfaites de l'information reçue } \\
\text { - insatisfaites du peu d'importance accordée } \\
\text { aux activités de loisirs ou d'établissement d'é- } \\
\text { change de services }\end{array}$ \\
\hline
\end{tabular}


Ces familles ont également émis des opinions sur les sujets discutés, franchissant ainsi une première étape de la résolution de problème, sans toutefois en arriver souvent à de l'interprétation ou à de la prescription de méthodes de changement.

Les groupes qui restent au niveau du simple partage d'idées et de vécu vivent quand même certains phénomènes associés à l'aide mutuelle, dont le soulagement qui découle du sentiment d'être tous dans le même bateau (Shulman, 1979). Le développement d'une aide mutuelle véritable exige un certain équilibre entre la demande et l'offre de soutien qui se produit dans le groupe, ainsi qu'une capacité des membres de surmonter les obstacles et de travailler ensemble (Shulman, 1979; Northen, 1969). L'utilisation plus consciente et plus constante du processus de groupe constituerait probablement un moyen d'atteindre un tel équilibre (Heap, 1988).

Les résultats quant à la formation des familles gardiennes indiquent que la majorité d'entre elles se sont senties aidées dans leur rôle de parent substitut et ont remarqué quelques modifications d'attitudes face aux familles utilisatrices. Les rencontres ont aussi contribué à un certain élargissement de leur perception du rôle à assumer et à un sentiment d'une plus grande implication dans le projet. Cependant, la plupart des familles gardiennes ont continué à définir ce rôle surtout par rapport aux enfants gardés plutôt qu'en termes d'aide aux familles utilisatrices. Seulement une minorité des familles affirment qu'elles ont changé certains comportements. Ce résultat $\mathrm{n}^{\prime}$ est toutefois pas surprenant : des recherches indiquent que des changements majeurs d'attitude ou de comportement se font plus lentement que les modifications de l'estime de soi car ils exigent la remise en question des habitudes, la réflexion sur les expériences passées et l'intégration de nouvelles connaissances (Home, 1980; Brundage et McKeracher, 1980).

Les familles gardiennes se sont montrées très satisfaites de leur groupe, tant par rapport au climat qui y prévalait qu'au contenu qui a été discuté. Elles se sentaient acceptées et à l'aise pour aborder des situations parfois difficiles rencontrées avec les enfants ou les parents. L'établissement d'un climat de confiance dans lequel les membres se sentent libres de prendre des risques est un prérequis à l'évolution d'un groupe vers un système d'aide mutuelle (Shulman, 1979). Cependant, les familles gardiennes auraient aimé se connaître davantage entre elles, ce qui laisse supposer que la confiance et l'intimité nécessaires à la pleine réalisation de l'aide mutuelle n'étaient pas encore suffisantes dans ce groupe. Au sujet du contenu du programme, les familles ont trouvé qu'il portait suffisamment sur le gardiennage, sans toutefois que les sujets abordés soient assez circonscrits pour leur indiquer des façons précises de procéder face à 
certains comportements difficiles de l'enfant tels que l'agressivité et le refus de la discipline.

Le groupe de FU avait comme objectif primaire de diminuer l'isolement des participantes, ce qui n'a pas été atteint : les familles ont indiqué peu de changement dans leur réseau suite aux rencontres. Les liens affectifs et l'entraide matérielle sous forme d'échange de services (par exemple, gardiennage de très courte durée) ne se sont pas réalisés. On peut expliquer ces résultats par le fait que peu de familles ont assisté à plus d'une ou deux rencontres et que les participantes ont varié d'une rencontre à l'autre.

Malgré leurs effets limités, les rencontres ont été jugées satisfaisantes, dans l'ensemble, par les participantes. Elles ont apprécié en particulier l'information reçue sur les ressources communautaires et sur certaines habiletés parentales telles que les premiers soins. Elles ont souligné aussi le climat d'encouragement mutuel et les occasions d'échange avec d'autres vivant des situations semblables. Toutefois, la plupart ont été insatisfaites du manque d'activités de loisirs. Ce résultat rejoint d'autres recherches sur les familles vulnérables : rares sont celles qui ont le temps, l'énergie et les ressources pour se permettre des loisirs, mais le besoin ne disparaît pas pour autant (Conseil national du Bien-être social, 1979). Les familles sont restées insatisfaites aussi face à l'absence d'échanges concrets tels que vêtements ou jouets. Compte tenu de leur situation socio-économique difficile, le besoin de cette forme de soutien matériel semble être, dans l'immédiat, plus important que le soutien affectif qui peut découler de la discussion.

En résumé, les deux groupes ont exprimé de la satisfaction quant au climat qui régnait lors des rencontres et quant aux thèmes abordés; des membres auraient toutefois aimé que certains aspects des programmes soient plus développés. Dans les deux groupes, il y a eu atteinte partielle des objectifs. Nous explorons, en conclusion, certaines pistes susceptibles de mener à un fonctionnement plus efficace des groupes et à une atteinte plus complète de leurs objectifs.

\section{Discussion et conclusion}

Les résultats de cette étude soulèvent différentes questions. L'une d'elles concerne les FU: quelles sont les façons les plus appropriées de les rejoindre et de les motiver à participer à un groupe ? Une deuxième question se pose pour les deux groupes: quel modèle (ou combinaison de modèles) est le mieux adapté aux objectifs visés ? Une troisième a trait à l'autonomie, au dévelop- 
pement du leadership et à la prise en charge dans le groupe des FG : quelle est la meilleure façon d'assurer soutien, encadrement et formation aux leaders non professionnels en émergence dans le groupe pour qu'ils y exercent leurs habiletés tout en favorisant la prise en charge et l'autonomie chez les membres ?

Plusieurs praticiens et praticiennes, auteurs et auteures constatent que les individus qui manquent de confiance ou d'habiletés sociales se joignent rarement aux groupes et lorsqu'ils le font, ils abandonnent en cours de route ou ne s'y impliquent pas (Gottlieb, 1985; Breton, 1979, 1985; Shulman, 1988). Les FU présentent des caractéristiques d'une clientèle difficile à rejoindre. La majorité d'entre elles n'ont pas accepté l'offre de service de groupe; certaines, qui avaient pourtant répondu positivement à l'invitation, ne se sont pas présentées aux rencontres et celles qui sont venues s'y sont peu impliquées (Breton, 1985). Deux aspects interreliés doivent alors être considérés : comment atteindre cette clientèle pour l'intéresser au groupe et comment la motiver à participer lorsqu'elle assiste aux réunions.

Breton (1985) souligne que les personnes qui ne répondent pas aux offres de service sont souvent convaincues que l'expérience ne vaut pas l'investissement de temps et d'énergie requis. Celles qui ont accepté verbalement de venir sans y donner suite ont peut-être hésité à dire non à une personne en position d'autorité ou se sont senties trop fatiguées pour affronter des obstacles matériels tels transport, conditions atmosphériques ou autres. La peur du risque ou d'un échec peut également entrer en ligne de compte (Breton, 1985). Les clientèles difficiles à rejoindre se sentent souvent impuissantes, sans espoir de modifier leur situation mais, en même temps, elles ont besoin d'être acceptées par leurs pairs (Shulman, 1988).

Différentes stratégies peuvent alors être envisagées. II faudrait, entre autres, interpréter clairement aux familles les objectifs des rencontres et démystifier le fonctionnement des groupes de façon à diminuer la peur ou la gêne des membres potentiels (Shulman, 1988). Des mesures concrètes facilitant l'accès aux rencontres peuvent également êtres utiles, par exemple : garderie sur place, moyen de transport (Breton, 1985; Heap, 1988). L'établissement d'un climat acceptant et chaleureux, tant dans les contacts pré-groupe que lors des réunions, peut aussi diminuer le sentiment de crainte provoqué par les groupes (Shulman, 1988). S'assurer que les femmes puissent participer aux décisions concernant l'organisation et le fonctionnement du groupe peut également aider à contrer le sentiment d'impuissance (Shulman, 1988). Une nuance s'impose toutefois : des défis optimaux doivent être présentés aux participantes pour les inciter à s'impliquer et à se développer mais sans outrepasser leurs 
capacités (Breton, 1985). En somme, il faut offrir une expérience attirante, non menaçante, tout en faisant preuve d'attentes réalistes.

Nous avons constaté que le groupe de FU est demeuré à l'étape de rassemblement (Henry, 1981); il présente même plusieurs caractéristiques d'une collectivité, forme intermédiaire entre l'agrégat et le groupe (Lang, 1986). Plusieurs participantes ont, en effet, démontré peu d'intérêt à s'impliquer dans le groupe et à y développer des liens forts entre membres. Elles ont préféré s'en tenir à des liens faibles (Shapiro, 1986). Pour certaines de ces personnes difficiles à rejoindre, on ne peut pas s'attendre à ce qu'elles s'engagent au-delà d'une telle collectivité. Celle-ci peut leur être utile, dans un premier temps, comme moyen de briser l'isolement et de s'offrir une activité de loisir. De plus, une collectivité peut être utilisée de façon délibérée avec des individus qui manquent $d^{\prime}$ habiletés à entrer en communication et à développer des liens avec d'autres, de façon à offrir une occasion continue de pratiquer ces habiletés (Lang, 1986 : 29-30).

Ceci nous amène à la deuxième question qui ressort de la recherche : quelle est l'orientation théorique et pratique la plus appropriée pour chacun des groupes? Au sujet des FU, nous avons remarqué que malgré les objectifs de socialisation poursuivis, ce groupe correspondait, en réalité, à un groupe d'information "café rencontre ". Étant donné que ce type de groupe exige peu d'implication des participantes, il peut être un moyen efficace d'offrir un soutien limité et un lieu de rencontre aux familles qui préfèrent s'en tenir à cette forme d'expérience. II faut toutefois offrir un programme attirant et amusant (Craig, 1988). On pourrait prévoir, à titre d'exemple, des réunions mensuelles comportant deux parties : I'une axée sur la socialisation informelle, incluant un goûter, et l'autre, plus structurée, offrant parfois de l'information (films, invités), parfois des activités de loisir. Un tel groupe pourrait se réunir mensuellement, à un moment prédéterminé, animé par des aidantes non professionnelles; ces dernières devraient bénéficier de l'encadrement et du soutien de travailleuses et travailleurs sociaux de groupe lorsqu'elles en ressentent le besoin et particulièrement lors des premières rencontres. Certaines personnes se contenteront d'assister à ces rencontres lorsque le programme les intéressera.

Pour d'autres, la participation à cette collectivité peut servir de contexte à risque limité pour se préparer à devenir membre d'un groupe (Lang, 1986). On pourrait, par exemple, offrir aux familles utilisatrices qui s'y intéressent un groupe de socialisation qui vise des objectifs d'intégration sociale et d'acquisition d'habiletés sociales (Toseland et Rivas, 1984). Un tel groupe, avec un membership semiouvert à noyau stable, auquel s'ajoutent des membres de temps en temps, pourrait se réunir aux quinze jours afin de favoriser une 
certaine cohésion entre membres. Alors que la collectivité a peu de structures et n'exige pas la présence d'un travailleur social ou d'une travailleuse sociale (autrement que dans le rôle de consultant), le groupe proposé pourrait s'inspirer du modèle à buts sociaux et bénéficier d'un leadership professionnel afin de favoriser éventuellement une plus grande participation des membres dans les prises de décisions et les tâches du groupe (Home et Darveau-Fournier, 1980). Ce processus risque toutefois d'être très long dans un groupe peu autonome (Lang, 1972). Comme le disent Heap (1988) et Breton $(1979,1985)$, il ne faut pas avoir peur, au début du groupe, de rester une "personne centrale» et de faire des démarches pour les membres surtout lorsque ceux-ci ont des habitudes de consommation de services ou ont peu d'expérience de groupe.

La planification des premières réunions devrait inclure des activités qui laissent suffisamment de place aux membres pour qu'ils puissent échanger et prendre certains risques, en tenant compte de leur capacité du moment (Henry, 1981). L'ensemble du programme doit aussi être choisi en tenant compte des capacités émotives des membres potentiels de s'engager et de communiquer, ainsi que des intérêts exprimés.

L'établissement d'un contrat clair constitue une stratégie à privilégier : alors que la collectivité offre des loisirs comme activités de détente et de contact, le groupe de socialisation s'en sert aussi comme moyen d'atteindre d'autres objectifs. Par exemple, des activités de loisir et d'échange de services (vêtements, recettes, etc.) peuvent divertir et aider les membres tout en facilitant leur engagement, dans la tradition du modèle à buts sociaux. L'utilisation explicite d'activités de loisir lors d'une première expérience de groupe a réussi, dans des centres de femmes entre autres, à donner le goût aux membres de s'engager dans des groupes qui demandent plus d'implication (Gingras, 1983; Home, 1980).

Considérant les exigences de rôles de parents substituts et d'aidantes non professionnelles confiés aux FG, de même que les objectifs de soutien et de formation visés par leur groupe, on peut croire qu'une combinaison du modèle de réciprocité/soutien (Shulman, 1979; Rosenburg, 1984) et de celui d'éducation structurée (Papell et Rothman, 1983) aiderait à rejoindre les besoins des membres. Les FG rencontrent divers problèmes concrets dans l'exercice quotidien du gardiennage; elles veulent des trucs ou façons de procéder pour surmonter les difficultés ou les défis posés tant par les enfants gardés que par les parents.

Le modèle d'éducation structurée peut fournir une contribution pertinente à ce groupe en suggérant des stratégies de nature à permettre des apprentissages particuliers. On peut penser, entre 
autres, à des exercices visant à développer des habiletés de communication avec les enfants ou les parents, à des activités systématiques (exposés, films, etc.) en vue d'acquérir des connaissances sur le développement de l'enfant ou sur certains comportements problématiques.

Toutefois, le simple fait de réunir des individus ne garantit nullement qu'ils vont réussir à travailler vraiment ensemble. Il est donc important d'aider les membres à dépasser la simple discussion et à développer des habiletés de résolution de problème. Les sujets traités devraient alors être centrés sur des aspects précis, et les membres, aidés à présenter des situations concrètes et à y trouver des solutions (Gitterman et Shulman, 1986).

La dernière question qui porte sur l'autonomie, le leadership et la prise en charge est sans doute la plus difficile à traiter, tant au niveau théorique que pratique. Plusieurs auteurs et auteures ont identifié les difficultés que soulève cette question (Alary et al., 1988; Lewis, 1983; Home, 1983) sans toutefois s'entendre sur la meilleure façon d'y répondre. $\mathrm{D}^{\prime}$ une part, le modèle du courant central suggère que l'intervenant ou l'intervenante devrait réduire son implication à mesure que l'autonomie du groupe croît, tout en tenant compte du niveau initial d'autonomie du groupe (Lang, 1972; Home et DarveauFournier, 1980; Henry, 1981). D'autre part, certains groupes populaires et groupes d'entraide préconisent plutôt la remise la plus complète possible des responsabilités aux membres dès le début du groupe dans le but de faciliter leur propre prise en charge. On peut alors se demander quelle est la formule la plus appropriée pour un groupe semi-autonome tel que celui des FG. Faut-il adopter dès le début un rôle périphérique afin de favoriser l'autonomie du groupe ou est-il préférable d'encadrer et de former d'abord des leaders afin de les aider à bien remplir leur rôle?

Compte tenu de l'importance accordée à la prise en charge du projet par les gens du milieu, les chercheuses ont opté pour une formule mixte. D'une part, elles ont occupé une position périphérique par rapport à l'ensemble du groupe (Henry, 1981); d'autre part, elles ont accordé beaucoup d'importance à la formation des leaders (Klein, 1972). L'expérience permet de conclure que le fait de laisser beaucoup de responsabilités au groupe comporte certains avantages, par exemple le fait que cette option offre aux membres l'occasion d'expérimenter des habiletés nouvelles et d'augmenter leur estime d'eux-mêmes. Par contre, pour les membres moins familiers avec les groupes, une prise de responsabilités trop hâtive peut provoquer de l'insécurité, des interventions plus ou moins appropriées et parfois même de la démotivation. II ne semble pas y avoir de réponse définitive à cette question. II faut considérer les 
avantages et les inconvénients des deux options tout en tenant compte de la réalité du milieu concerné.

En résumé, l'analyse comparative des deux interventions permet de constater que les deux groupes étaient satisfaits et du climat et du contenu des rencontres. Toutefois, le groupe des FG a mieux réussi ses objectifs que celui des FU. II serait possible de faciliter une atteinte plus complète des objectifs en réexaminant les moyens de rejoindre une telle population et le choix le plus approprié de modèles théoriques sous-jacents à l'intervention.

\section{Notes}

* Article préparé à la suite d'une présentation au symposium de Montréal.

${ }^{1}$ Ce projet a été mené et évalué durant trois ans (de septembre 1984 à 1987) grâce à l'appui du Programme de Subventions nationales au Bien-être social.

${ }^{2}$ Afin d'alléger le texte, nous utiliserons FG pour familles gardiennes et FU pour familles utilisatrices.

${ }^{3}$ Pour des informations plus complètes sur le devis et les instruments d'évaluation ainsi que sur le déroulement et le fonctionnement du projet, on pourra consulter : Le soutien aux familles... Une formule à redécouvrir. Rapport final d'évaluation du projet Familles-gardiennes de la Basse-Ville, à soumettre par les auteures de l'article à Santé et Bien-être social Canada à l'automne 1990.

\section{Références}

Alary, J., J. Beausoleil, M.C. Guédon, C. Larivière et R. Mayer (1988). Solidarités. Pratiques de recherche-action et de prise en charge par le milieu. Montréal : Boréal.

AUERBACH, Aline B. (1976). L'éducation des parents par le groupe: principes de base. Traduction de Lise Fournier et Gisèle Turcot du chap. 1: Parents learn through discussion: Principles and practices of parent group education. New York : John Wiley \& Sons (1968).

BRETON, M. (1979). "Nurturing abused and abusive mothers : The hairdressing group ", Social Work with Groups, vol. 2, n² 2 : 161-173.

BRETON, M. (1985). "Reaching and engaging people: Issues and practice principles", Social Work with Groups, vol. 8, n० 3 : 7-21.

Brundage, D. et D. MCKeracher (1980). Adult learning principles and their application to programme planning. Toronto : Minister of Education, Ontario.

Comité de la santé mentale du Québec (1985). La santé mentale des enfants et des adolescents: vers une approche plus globale. Gouvernement du Québec : Division des publications gouvernementales.

Conseil national du Bien-être social (1979). Pour améliorer le sort des enfants. Rapport sur le système d'aide à l'enfance du Canada. Ottawa : gouvernement du Canada. 
COYLE, G.L. (1959). "Some basic assumptions about social group work ", Social work curriculum study, vol. XI : The Social Group Work Method : 88-105. New York, Council on Social Work Education.

CRAIG, R. (1988). "Structured activities with adolescent boys ", Groupwork, vol. 1, $\mathrm{n}^{\circ} 1:$ 48-59.

DarVeau-Fournier, L. et A. Home (sous presse). "Advancing community group work theory through action-research ", à paraître dans un numéro spécial de Social Work with Groups.

GARBARINO, J. (1986). "Where does social support fit into optimizing human development and preventing dysfunction?", British Journal of Social Work, vol. 16, Supplément : 23-37.

GARBARINO, J. et D. SHERMAN (1980). "High-risk neighborhoods and high-risk families : The human ecology of child maltreatment ", Child Development, vol. 5, $\mathrm{n}^{\circ} 1:$ 188-198.

Gingras, P. (1983). "Intervention auprès de femmes de classe populaire ", Service social, vol. 32, $\mathrm{n}^{\text {os }} 1-2: 89-100$.

Gitterman, A. et L. Shulman (1986). Mutual aid groups and the life cycle. Itasca, III. : Peacock.

GotтLев, B.H. (1985). "Combining lay and professional resources to promote human welfare : Prospects and tensions »: 59-78, dans J.A. Yoder (éd.), Support networks in a caring community. Dordrecht, Netherlands : Martinus Nijhoff.

GottleB, B.H. (1983). Social support strategies. Beverly Hills, CA : Sage.

GOTLIEB, B.H. (1986). "Using social support to protect and promote health ", Journal of Primary Prevention, vol. 8, $\mathrm{n}^{\text {os }} 1-2:$ 49-70.

HARTFORD, M. (1971). Groups in social work: Application of small group theory and research to social work practice. New York : Columbia University Press.

HeAP, K. (1988). "The worker and the group process : A dilemma revisited", Groupwork, vol. 1, $\mathrm{n}^{\circ} 1: 17-29$.

HenRY, S. (1981). Group skills in social work : A four dimensional approach. Itasca, III. : Peacock.

HOME, A. (1980). "Étude exploratoire de quatre types de changements survenus chez les membres de groupes féminins de conscientisation ", Service social, vol. 29, $\mathrm{n}^{\text {os }} 1-2: 152-181$.

HOME, A. (1985). "Intervention with groups", dans S. Yelaja, An introduction to social work practice in Canada. Toronto : Prentice-Hall.

Home, A. (1983). "Les femmes et les groupes de changement social ", Service social, vol. 32, $\mathrm{n}^{\text {os }} 1-2: 50-79$.

Home, A. et L. Darveau-Fournier (1980). "La spécificité du service social des groupes ", Service social, vol. 29, $\mathrm{n}^{\text {os }} 1-2$ : 16-31.

KLEIN, A. (1972). Effective groupwork. New York: Association Press.

LANG, N. (1972). "A broad range model of practice in the social work group ", Social Service Review, vol. 46, $\mathrm{n}^{\circ} 1$ : 76-82.

LANG, N. (1986). "Collectivity in social group work ", Social Work with Groups, vol. 9, $\mathrm{n}^{\circ} 4:$ 7-32.

LEWIS, E. (1983). "Le service social des groupes dans la vie communautaire", Service social, vol. $32, \mathrm{n}^{\text {os }} 1-2: 32-49$. 
Morrison, W., G. Pace, M. Sehl et H. Smith (1986). "Single mothers in Canada: An analysis», Revue canadienne de santé mentale communautaire, vol. $5, \mathrm{n}^{\circ} 2: 37-47$.

NORTHEN, H. (1969). Social work with groups. New York : Columbia University Press.

PAPell, C. et B. Rothman (1983). "Le modèle du courant central du service social des groupes en parallèle avec la psychothérapie et l'approche de groupe structuré ", Service social, vol. 32, $\mathrm{n}^{\text {os }} 1-2: 11-31$.

ROSENBERG, P.P. (1984). "Support groups : A special therapeutic entity », Small Group Behavior, vol. 15, $\mathrm{n}^{\circ} 2$ : 173-186.

RutMAN, L. (1982). "La recherche formative et l'évaluabilité d'un programme " : 65-79, dans R. Lecomte et L. Rutman (éd.), Introduction aux méthodes de recherche évaluative. Québec : P.U.L.

SCHOPLER, J.H. et M.J. GALINSKY (1984). "Meeting practice needs : Conceptualizing the open-ended group ", Social Work with Groups, vol. 7, n ${ }^{\circ} 2$ : 3-21.

SeaburG, J. (1985). "Sampling »: 133-148, dans R. Jr. Grinnell (éd.), Social work research evaluation ( $2^{\mathrm{e}}$ édition). Itasca, III. : Peacock.

SHAPIRO, B. (1986). "The weak tie collectivity : A network perspective ", Social Work with Groups, vol. 9, n 4 : 113-125.

SHULMAN, L. (1988). "Group work practice with hard to reach clients: A modality of choice", Groupwork, vol. 1, n ${ }^{\circ} 1: 5-16$.

SHuLman, L. (1979). The skills of helping individuals and groups. Itasca, III. : Peacock.

TOSELAND, R. et D. Rivas (1984). An introduction to group work practice. New York et London : MacMillan. 